\title{
TOPOLOGICAL UNIQUENESS OF NEGATIVELY CURVED SURFACES
}

\author{
HSUNGROW CHAN
}

\begin{abstract}
In this paper we consider complete, noncompact, negatively curved surfaces that are twice continuously differentiably embedded in Euclidean threespace, showing that if such surfaces have square integrable second fundamental form, then their topology must, by the index method, be an annulus. We then show how this relates to some minimal surface theorems and has a corollary on minimal surfaces with finite total curvature. In addition, we discuss, by the index method, the relation between the topology and asymptotic curves. Finally, we apply the results yielded to the problem of isometrical immersions into Euclidean three-space of black hole models.
\end{abstract}

\section{$\S 1$. Introduction}

Geometers have been studying minimal surfaces for many years. Their studies have extended in many different directions. Here, we generalize according to classical surface theory. We examine which minimal surface theorems with finite total curvature $\left(\int|K|<\infty\right)$ continue to hold true if the vanishing mean curvature $H \equiv 0$ is changed to $\int H^{2}<\infty$. Because the Gauss curvature $K$ of minimal surfaces is nonpositive, the generalization will keep the curvature nonpositive. Let $|B|$ be the length of the second fundamental form, $|B|^{2}=4|H|^{2}-2 K$. Minimal surfaces with finite total curvature belong to the subset of nonpositively curved surfaces with square integrable second fundamental form $\int|B|^{2}<\infty$. Thus, under different topological assumptions, we have several results which generalize the Bernstein's minimal graph theorem; an entire minimal graph in $R^{3}$ must be a plane. For example, we showed that if $M$ is a complete, simply connected, nonpositively curved surface embedded in $R^{3}$ with $\int|B|^{2}<\infty$, then it must be a plane [3]. As another example, we proved that if $M$ is a complete one-ended,

Received June 6, 2008. Revised December 16, 2009. Accepted December 17, 2009.

2000 Mathematics Subject Classification. 0240G, 0240M, 0240E, 0470B.

This work was supported by the National Science Council, Taiwan.

(C) 2010 by The Editorial Board of the Nagoya Mathematical Journal 
nonpositively curved surface immersed in $R^{3}$ with the end embedded near infinity and $\int|B|^{2}<\infty$, then it must lie between two parallel planes [4].

In this paper we constrain the Gauss curvature from nonpositivity to negativity and consider the condition of $\chi(M) \leq 0$. From the above examples, a negatively curved surface in $R^{3}$ with $\int|B|^{2}<\infty$ and $\chi(M)=1$ cannot exist, nor can a negatively curved surface in $R^{3}$ with one embedded end and $\int|B|^{2}<\infty$. The main theorem of this paper is as follows.

THEOREM 1. If $M$ is a complete negatively curved surface $C^{2}$ isometrically embedded in $R^{3}$ with $\int|B|^{2}<\infty$, then $\chi(M)=0$.

To a degree, Theorem 1 relates to some minimal surface theorems and a minimal surface conjecture with finite total curvature.

TheOREM 2 (Schoen [11]). A properly embedded minimal surface with $\int|K|<\infty$ and two embedded ends must be a catenoid.

Theorem 3 (Lopez and Ros [8]). The plane and the catenoid are the only embedded, complete, minimal surfaces in $R^{3}$ with $\int|K|<\infty$ and genus zero in $R^{3}$.

Conjecture 4 (Meeks and Perez, see [5]). A complete, embedded, minimal surface in $R^{3}$ with negative curvature must be a catenoid, a helicoid, or a scherk 1- or 2-periodic surface in particular; there exist no minimal examples of complete, embedded, negatively curved surfaces with a negative Euler characteristic in $R^{3}$.

The main object of Theorems 2 and 3 and Conjecture 4 is to show the uniqueness of the catenoid among the minimal surfaces with finite total curvature. The Gauss curvature of a catenoid is negative. It is thus natural to focus on the negatively curved surfaces with $\int H^{2}<\infty$ and $\int|K|<$ $\infty$. When $H \equiv 0$ is changed to $\int H^{2}<\infty$, the uniqueness of the catenoid in minimal surfaces with finite total curvature is changed to topological uniqueness $(\chi(M)=0)$ in negatively curved surfaces with $\int|B|^{2}<\infty$. We can show the following as an application of Theorem 1.

Conjecture 5. The only complete embedded minimal surface with finite total curvature and without umbilic points are the plane and the catenoid.

The remainder of this paper is organized as follows. In Section 2 we discuss the index method and give several examples of its application. The proof of Theorem 1 is given in Section 3, and in Section 4 we apply the index 
method to the orthogonal projections of the asymptotic curves. Finally, in Section 5 we apply Theorem 1 to the problem of isometrical immersions into Euclidean three-space of black hole models.

\section{$\S 2$. Index method}

In 1987, White [14] proved that if $M$ is a complete, oriented, connected, nonpositively curved surface $C^{2}$-immersed in $R^{3}$ with $\int|B|^{2}<\infty$, then the Gauss map extends continuously to one point near infinity, and $M$ is properly immersed near infinity. Thus, the complement of a big compact set of a negatively curved embedded surface $M$ in $R^{3}$ with $\int|B|^{2}<\infty$ is a union of a finite number of graph ends $p_{1}, \ldots, p_{n}$. Let $C$ be a continuous nonvanishing line field on $M$. Let $\gamma: I \rightarrow M \subset R^{3}$ be a simple closed curve parameterized by arc length $s \in I$ looping around the graph end $p_{i}$. Let $T(s)=\gamma^{\prime}(s)$ be the unit tangent vector of $\gamma$. Then $C$ is the continuous line field on $M$ along $\gamma(s)$. Let $\alpha$ be the angle from the vectors $T(s)$ to $\vec{C}(s)$, where $\vec{C}(s)$ is a continuous vector field along $\gamma$ representing $C$. Let $\Delta \alpha$ be the increment of $\alpha(s)$ as $\gamma$ is traversed once around in the positive sense. The index of a graph end $p_{i}$ corresponding to the line field $C$ is defined by

$$
L\left(p_{i}\right)=1 \pm \frac{\Delta \alpha}{\pi}
$$

Let $(0,0,1)$ be the $z$-direction of the plane $P$ for the graph end and the north pole of the unit sphere. Define $\gamma$ to be a clockwise loop corresponding to $(0,0,1)$. There are two cases for the images of a Gauss map. In case 1 , the image of $\gamma$ is clockwise, and in case 2 it is counterclockwise corresponding to $(0,0,1)$. In case 1 , define $L\left(p_{i}\right)=1-\Delta \alpha / \pi$, and in case 2 , define $L\left(p_{i}\right)=1+\Delta \alpha / \pi$. The index of a graph end corresponding to the line field is independent of loops chosen.

There are four natural and well-defined line fields on a negatively curved surface $M$ in $R^{3}$. Two are the principal directions, and the other two are the asymptotic directions. Let $\mathcal{A}_{1}$ and $\mathcal{A}_{2}$ be the two families of asymptotic lines on $M$. We define the index of $\mathcal{A}_{j}, j=1,2$, and denote $L_{j}\left(p_{i}\right)$ in a manner analogous to defining indices of vector fields about singularities (or zero). It is clear that $L_{1}\left(p_{i}\right)=L_{2}\left(p_{i}\right)$. The index of an end is independent of these four lines chosen.

Verner [13] found the following Poincare index formula for complete, noncompact surfaces with $K \leq 0$ and $\int|K|<\infty$ and used it to classify the 
number of possible horn and bowl ends for complete, connected, smoothly embedded surfaces in $R^{3}$.

THEOREM 6 (Verner [13]). If $M$ is a complete, noncompact, $C^{2}$ negatively curved surface with $\int|K|<\infty$, then

$$
2 \chi(M)=\sum_{i=1}^{n}\left(L\left(p_{i}\right)-1\right),
$$

where $L\left(p_{i}\right)$ is the index of the end $U_{i}$ corresponding to an asymptotic line field $C$.

Since the Gauss map extends continuously to one point near the infinity of each graph end, we define a map from the line field of the end to the line field of a neighborhood of a point on the sphere $S^{2}$. This maps the infinity of an end to a point of $S^{2}$. We can then adapt the definition for elliptic, parabolic, and hyperbolic sectors (Hartman [7, page 166]). Let $n_{h}$ be the number of hyperbolic sectors, and let $n_{e}$ be the number of elliptic sectors around a Jordan curve near infinity or the singularity. We apply Hartman's formula to compute the index of a vector field, so $L\left(p_{i}\right)=1+n_{e}-n_{h}$. It is clear that two definitions of $L\left(p_{i}\right)$ are consistent in the proof of Verner's theorem, and the index is independent of the homotopic regular, simple closed curves looping around the end.

ExAmPle 1. The Gauss curvature of graph $M_{1}=\{(x, y, x y)\}$ is negative, and the projections of asymptotic lines to the $x y$-plane are $\{x=$ constant $\}$ and $\{y=$ constant $\}$. There are two elliptic sectors at infinity. The index of this graph end, then, is $L\left(p_{1}\right)=1+2=3$. Let $\gamma: I \rightarrow M_{1}$ be a simple closed circle. The increment of angle $\alpha$ as $\gamma$ traversed is $2 \pi$. The Gauss image of clockwise $\gamma$ is counterclockwise, so

$$
L\left(p_{1}\right)=1+\frac{2 \pi}{\pi}=3 .
$$

By Theorem 6 , we have $2 \chi\left(M_{1}\right)=L\left(p_{1}\right)-1=2$. Therefore, $\chi\left(M_{1}\right)=1$.

EXAMPLE 2. Let $M_{\text {rev }}$ be a surface of revolution which is revolving a graph of a convex function from $R$ to $R$, in the $x y$-plane, about the $x$-axis. The Gauss curvature, then, of $M_{\text {rev }}$ is negative. There exists a shortest closed geodesic curve $\gamma_{g}$ in the throat part, and it divides the surface into two ends: $p_{1}$ and $p_{2}$. The convexity and the circular symmetry force the 
asymptotic curves from one end to the other. Thus, there are no hyperbolic and elliptic sectors for each end; that is, $L\left(p_{i}\right)=1$. The increment of angle $\alpha$ as $\gamma$ is traversed is $0 . L\left(p_{i}\right)=1+0=1$. By Theorem 6 , we have

$$
2 \chi\left(M_{\text {rev }}\right)=\left(L\left(p_{1}\right)-1\right)+\left(L\left(p_{2}\right)-1\right)=0+0=0 .
$$

Therefore, $\chi\left(M_{\text {rev }}\right)=0$.

ExAmple 3. An important example is Hadamard's surface. A hyperboloid of one sheet $M_{\text {hyp }}$ can be considered a solution described by the equation $x^{2}+y^{2}-z^{2}-1=0$ in $R^{3}$, or a revolving hyperboloid by rotation of the curve $\left(x, 0, \sqrt{x^{2}+1}\right)$ about the $x$-axis. $M_{h y p}$ is one kind of $M_{r e v}$. Its Gauss image does not converge to one point at infinity. It may also be parameterized as a ruled surface on which

(1) $\Psi(v, \theta)=\alpha(\theta)+v\left((0,0,1)+\alpha^{\prime}(\theta)\right)=(\cos \theta, \sin \theta, 0)+v(-\sin \theta, \cos \theta, 1)$,

where $\alpha(\theta)$ is a circle on the $x y$-plane and $\alpha^{\prime}(\theta)$ is the tangent vector. By replacing $(0,0,1)-\alpha^{\prime}(\theta)$, we obtain the same surfaces. This shows that a revolving hyperboloid has two sets of mappings. The straight lines $(0,0,1) \pm$ $\alpha^{\prime}(\theta)$ are the two families of asymptotic lines. Clearly, $\chi\left(M_{h y p}\right)=0$.

ExAmple 4. Let $U$ and $V$ be two hyperboloids of one sheet as follows:

$$
\begin{aligned}
& U: x^{2}+(y-2)^{2}=z^{2}+1, \\
& V: x^{2}+(y+2)^{2}=z^{2}+1 .
\end{aligned}
$$

Hadamard [6] merged $U$ and $V$ to get the union surface $M_{h a d}^{2}$. He smoothed the edge and proved that the union was a negatively curved surface which was homeomorphic to a two-points-punctured torus. Thus, $\chi\left(M_{\text {had }}\right)=-2$.

On the upper plane $\{y=0\}$, the intersection curve $\left\{z^{2}-x^{2}=3\right\}$ of $M_{h a d}^{2}$ is an opening, upward hyperbola. The straight asymptotic lines from two surfaces meet along the intersection curve, and they form two hyperbolic sectors from two sides for the upper end. Parts of the asymptotic lines come from the lower end, meet the intersection curve, and bounce back to the lower end. There is no elliptic sector for the end. Thus $L\left(p_{i}\right)=1-2=-1$. The increment of angle $\alpha$ as $\gamma$ traversed is $2 \pi$. The Gauss image of clockwise $\gamma$ is clockwise, so

$$
L\left(p_{1}\right)=1-\frac{2 \pi}{\pi}=-1 .
$$


By Theorem 6, we have $2 \chi\left(M_{h a d}^{2}\right)=\left(L\left(p_{1}\right)-1\right)+\left(L\left(p_{2}\right)-1\right)=-2-2=-4$. Therefore, $\chi\left(M_{\text {had }}^{2}\right)=-2$. In general, for the union of $n$ hyperboloids of one sheet,

$$
\chi\left(M_{h a d}^{n}\right)=-2(n-1) .
$$

In [2], we proved that Hadamard's method cannot apply to merge two surfaces of revolution with their Gauss image converging to one point at each infinity.

\section{§3. Proof of Theorem 1}

We will prove that the index of each graph end of $M$ is 1 . Then, by Theorem $6,2 \chi(M)=\sum_{i=1}^{n}\left(L\left(p_{i}\right)-1\right)=0$, which finishes the proof of Theorem 1 . Let $\gamma: I \rightarrow M \subset R^{3}$ be a simple, closed curve parameterized by arc length $s \in I$ looping around the graph end $p_{i}$. Let $\mathcal{N}(s)$ be the unit normal vector to the surface at the point $\gamma(s) \in M$, and let $b(s)=T(s) \times \mathcal{N}(s)$ be the unit conormal of $\gamma$. By differentiating the vectors $\{T, b, \mathcal{N}\}$, we have the Frenet formula adapted to the surface:

$$
\left\{\begin{array}{l}
T^{\prime}=k_{g} b+k_{n} \mathcal{N}, \\
b^{\prime}=-k_{g} T+\tau_{g} \mathcal{N}, \\
\mathcal{N}^{\prime}=-k_{n} T-\tau_{g} b,
\end{array}\right.
$$

where $\tau_{g}$ is the torsion of $\gamma, k_{g}$ is the geodesic curvature of $\gamma$, and $k_{n}$ is the conormal curvature of $\gamma$. Let $g$ be the Gauss map, and let $k_{g}^{*}$ be the geodesic curvature of the image $g(\gamma(s))$ on $S^{2}$. Let $\rho$ be the arc length along the image $g(\gamma(s))$ on $S^{2}$. Then $d \rho= \pm \sqrt{k_{n}^{2}+\tau_{g}^{2}} d s$, where the sign of $d \rho$ depends on orientations of $\gamma(s)$ and $g(\gamma(s))$ in $R^{3}$.

Since $K<0, \mathcal{N}^{\prime}(s) \neq 0$ at every point on $\gamma$, and $\mathcal{N}^{\prime}(s)$ is a continuous nonvanishing vector field along $\gamma$. Let $\alpha(s)$ be the angle between $T(s)$ and $\mathcal{N}^{\prime}(s)$, and let $\Delta \alpha$ be the increments of $\alpha(s)$ along $\gamma(s)$ going around once. Since $\tan \alpha(s)=\tau_{g} / k_{n}$,

$$
\frac{d \alpha}{d s}=\frac{k_{n} \tau_{g}^{\prime}-k_{n}^{\prime} \tau_{g}}{k_{g}^{2}+\tau_{g}^{2}}
$$

Along $g(\gamma(s))$,

$$
k_{g}^{*}=\frac{\left|\left(\mathcal{N}(s), \mathcal{N}^{\prime}(s), \mathcal{N}^{\prime \prime}(s)\right)\right|}{\left|\mathcal{N}^{\prime}(s)\right|^{3}}=\frac{k_{g}}{\sqrt{k_{n}^{2}+\tau_{g}^{2}}}+\frac{k_{n} \tau_{n}^{\prime}-k_{n}^{\prime} \tau_{g}}{\left(k_{n}^{2}+\tau_{g}^{2}\right)^{3 / 2}} .
$$


Then, multiplying by $\sqrt{k_{n}^{2}+\tau_{g}^{2}}$ and taking the line integral of (3) along $\gamma(s)$, we have

$$
\int_{\gamma(s)} k_{g}^{*} \sqrt{k_{n}^{2}+\tau_{g}^{2}} d s= \pm \int_{g(\gamma(s))} k_{g}^{*} d \rho=\int_{\gamma(s)} k_{g} d s+\int_{\gamma(s)} \frac{k_{n} \tau_{g}^{\prime}-k_{n}^{\prime} \tau_{g}}{k_{n}^{2}+\tau_{g}^{2}} d s .
$$

By (2), the second integral is

$$
\int_{\gamma(s)} \frac{k_{n} \tau_{g}^{\prime}-k_{n}^{\prime} \tau_{g}}{k_{n}^{2}+\tau_{g}^{2}} d s=\int_{\gamma(s)} d \alpha=\Delta \alpha= \pm \pi\left(L\left(p_{i}\right)-1\right) .
$$

Thus, Verner proved the following lemma, which showed the relationship between the geodesic curvatures and the index of an end.

Lemma 7 [12]. Let $\gamma(s)$ be a closed $C^{3}$-curve on a $C^{3}$-surface $M$, which loops around an end $U$ and is parameterized by arc length. If $\mathcal{N}^{\prime}(s) \neq 0$ at every point on $\gamma$, then

$$
\pm \int_{g(\gamma(s))} k_{g}^{*} d \rho=\int_{\gamma(s)} k_{g} d s \pm \pi\left(L\left(p_{i}\right)-1\right),
$$

where the first sign of $d \rho$ depends on orientations of $\gamma(s)$ and $g(\gamma(s))$ and the second sign depends on the image of the Gauss map.

Now, we prove Theorem 1. By applying our theorem in [3], $M$ is not simply connected, so there is a shortest closed, geodesic curve $\gamma_{g}$ surrounding $p_{i}$ for each graph end. Let $\gamma_{i}$ be a sequence of the level circles extending to the infinity, which are homotopic to $\gamma_{g}$. Let $D_{i}$ be the region between $\gamma_{g}$ and $\gamma_{i}$. By the Gauss Bonnet theorem,

$$
\int_{D_{i}} K d A+\int_{\gamma_{i}} k_{g}+\int_{\gamma_{g}} k_{g}=2 \pi \chi\left(D_{i}\right)=0
$$

Then, $\int_{\gamma_{i}} k_{g}=-\int_{D_{i}} K d A$. Since the Gauss curvature is negative and the Gauss map is one-to-one and surjective, $\int_{\gamma_{i}} k_{g} \rightarrow 2 \pi$ as $i \rightarrow \infty$. On the Gauss image of $M_{\text {rev }}$, we have

$$
\int_{g\left(\gamma_{i}\right)} k_{g}^{*} d \rho=-\int_{D_{i}^{*}} K d A^{*}=-\operatorname{Area}\left(D_{i}^{*}\right),
$$

where $K=1$ for a unit sphere. When $i \rightarrow \infty, \int_{g\left(\gamma_{i}\right)} k_{g}^{*} \rightarrow-2 \pi$. Then, by Lemma $7, L\left(p_{i}\right)=1$ and

$$
\chi(M)=\frac{1}{2}\left(\sum_{i=1}^{n}\left(L\left(p_{i}\right)-1\right)\right)=0 .
$$


Therefore, there are exactly two ends for $M$, and its topology is unique. This completes the proof.

\section{§4. Orthogonal projection of the asymptotic curves}

In this section, we use the index method on the orthogonal projections of the asymptotic curves and get an inequality on the $\chi(M)$, where $M$ is a negatively curved surface. Brandt (see [10]) used the projection to show that there is no smooth asymptotic loop beginning and ending at the isolated parabolic point on a smooth surface with $K \leq 0$. Now, we apply Brandt's method to the graph of functions on an $x y$-plane and give a proof of nonexistence of smooth asymptotic loop at infinity of a graph end.

Consider a graph of a function $f(x, y)$ on a domain in $R^{2}$ with $K<0$. Let $\Theta_{i}(i=1,2)$ be the angle between the $x$-axis and the projection of the $i$ th asymptotic line on $(x, y)$-plane, which is determined up to a multiple of $\pi$. Define the map $\Phi$ from $(x, y)$-plane to $\left(x^{\prime}, y^{\prime}\right)$ by

$$
\Phi:(x, y) \mapsto\left(f_{y},-f_{x}\right) .
$$

Since $K<0$, $\operatorname{det}(d \Phi)<0$. Let $\Theta_{i}^{\prime}$ be the angle between the $x^{\prime}$-axis and the image of the $i$ th asymptotic line on the $x^{\prime} y^{\prime}$-plane.

LEMMA 8. $\Theta_{i}=\Theta_{i}^{\prime}$.

Proof. For a graph $(x, y, f(x, y))$, the first fundamental form is

$$
d s^{2}=\left(1+f_{x}^{2}\right) d x^{2}+2 f_{x} f_{y} d x d y+\left(1+f_{y}^{2}\right) d y^{2},
$$

and the second fundamental form is

$$
I I=\frac{1}{\sqrt{1+f_{x}^{2}+f_{y}^{2}}}\left(f_{x x} d x^{2}+2 f_{x y} d x d y+f_{y y} d y^{2}\right) .
$$

Let $(a, b)$ be the asymptotic line; then

$$
(a, b)\left(\begin{array}{ll}
f_{x x} & f_{x y} \\
f_{y x} & f_{y y}
\end{array}\right)\left(\begin{array}{l}
a \\
b
\end{array}\right)=0
$$

that is, $f_{x x} a^{2}+2 f_{x y} a b+f_{y y} b^{2}=0$. We are going to show that $d \Phi((a, b))$ is in the same direction as $(a, b)$. Now $(b,-a) \cdot(a, b)=0$. Also

$$
(b,-a) \cdot d \Phi((a, b))=(b,-a)\left(\begin{array}{cc}
f_{y x} & f_{y y} \\
-f_{x x} & -f_{x y}
\end{array}\right)\left(\begin{array}{l}
a \\
b
\end{array}\right)=0
$$


that is, $d \Phi((a, b))=c(a, b)$, where $c$ is a nonzero function. Therefore, $\Theta_{i}=$ $\Theta_{i}^{\prime}$, since $K<0$. This completes the proof.

We can assume that a local domain of a $C^{3}$ surface is given by a graph of a function $z(x, y)$ of class $C^{3}$ in a certain circle $x^{2}+y^{2}<a^{2}$. Assume that the surface is with $K \leq 0$ and each parabolic point $p_{0}$ is isolated. Brandt [10] defined an asymptotic loop as regular at a point $p_{0}$, if its tangent tends to a limiting position at $p_{0}$ on each of the two ends of the loop, where $p_{0}$ is an isolated parabolic point in the nonpositive curvature surface. Brandt [10] proved that if $U\left(p_{0}\right)$ of $p_{0}$ is of class $C^{1}$ and if $U\left(p_{0}\right) \backslash p_{0}$ is of class $C^{2}$, then in a sufficiently small neighborhood $U^{\prime}$ of $p_{0}$, asymptotic loops regular at $p_{0}$ do not exist.

Now, by modifying Brandt's proof, we can prove Lemma 10 using a similar argument on the graph end and under similar definitions of regular at infinity. Let $U$ be a graph end homeomorphic to a punctured disk in $R^{3}$ such that the Gauss map $g: U \rightarrow S^{2}$ converges to one point at infinity; that is, $\lim _{x \rightarrow p} g(x)=q, q \in S^{2}$. We can arrange by rotation so that $\lim _{x \rightarrow p} g(x)=(0,0,1)=P$. If there is no confusion, let $P$ be the $x y$-plane and the unit vector $(0,0,1)$. Let $\Pi: R^{3} \rightarrow P$ be the orthogonal projection. We consider the projection on $P$ from one family of asymptotic curves.

DeFinition 9. We call an asymptotic line regular at infinity if the tangent vector of the asymptotic line viewed in $R^{3}$ has a limit as $t \rightarrow \infty$. We call a graph end $U$ perfectly regular if the projections of asymptotic curves from one family near infinity satisfy the following.

(1) Any asymptotic curve tending to infinity is regular at infinity.

(2) There is a coordinate system near the infinity on a punctured disk so that the asymptotic lines field is given by a $C^{1}$ vector field, which vanishes at infinity.

Lemma 10. Let $U$ be a $C^{3}$ perfectly regular graph end in $R^{3}$ with $K<0$ and the Gauss map converging to one point at infinity. There is, then, no elliptic sector of asymptotic lines in $U$ that means asymptotic lines coming from, and going back to, infinity.

Proof. Assume that there is an elliptic sector for a graph end. That is to say, there is a $\gamma \in \mathcal{A}$ such that both ends of $\gamma$ regularly go to infinity. $\gamma$ cannot intersect itself, so $\gamma$ divides $R^{2}$ into two parts. Let $\gamma(s)$ go in a fixed direction. 
Let $\Phi(\gamma)$ be the image of $\gamma$ of the map $\Phi$. Since the Gauss map converges to one point at infinity, $\Phi(\gamma)$ is a closed loop beginning and ending at $(0,0)$ on the $x^{\prime} y^{\prime}$-plane.

For each $q^{\prime} \in \Phi(\gamma(s))$, let $O=(0,0)$, let $\phi^{\prime}$ be the angle from $\overline{O x^{\prime}}$ to $\overline{O q^{\prime}}$, and let $\beta^{\prime}$ be the angle from $\overline{O q^{\prime}}$ to the tangent vector $\left.\Phi(\gamma)^{\prime}\right|_{q}$. Let $\Delta \Theta^{\prime}, \Delta \beta^{\prime}$, and $\Delta \phi^{\prime}$ be the increments of the angle $\Theta^{\prime}, \beta^{\prime}$, and $\phi^{\prime}$ after a single positive circuit of $\Phi(\gamma)$. By smoothness of the asymptotic lines at infinity and the property of the map $\Phi$, we have

$$
\Delta \Theta^{\prime}=\Delta \beta^{\prime}+\Delta \phi^{\prime}, \quad \Delta \beta^{\prime}=\pi, \quad \Delta \phi^{\prime}>0 .
$$

For each $q \in \gamma(s)$, let $O=(0,0)$, let $\phi$ be the angle between $\overline{O x}$ and $\overline{O q}$, and let $\beta$ be the angle between $\overline{O q}$ and the vector $\left.\gamma^{\prime}\right|_{q}$. Let $\Delta \Theta, \Delta \beta$, and $\Delta \phi$ be the increments of the angle $\Theta, \beta$, and $\phi$ after a single positive circuit of $\gamma$. By smoothness of the loop $\gamma$ at infinity, we have

$$
\Delta \Theta=\Delta \beta+\Delta \phi .
$$

By Lemma 7 and (4), we have $\Delta \Theta=\Delta \Theta^{\prime}>\pi$.

Let $N$ be the degree of the Gauss map of $U$. We consider the mapping $\Psi:\left(x^{\prime}, y^{\prime}\right) \rightarrow\left(x^{\prime \prime}, y^{\prime \prime}\right)$ defined by

$$
x^{\prime \prime}+i y^{\prime \prime}=\sqrt[|N|]{x^{\prime}+i y^{\prime}},
$$

where $i=\sqrt{-1}$. The composition of the mapping $\Psi \circ \Phi$ gives a homeomorphism $(x, y) \rightarrow\left(x^{\prime \prime}, y^{\prime \prime}\right)$ of degree -1 , so $\gamma$ is mapped into a curve $\Psi(\Phi(\gamma))$ traversed in the opposite direction.

On the $\left(x^{\prime \prime}, y^{\prime \prime}\right)$-planes, we introduce the angles to $\phi^{\prime \prime}, \beta^{\prime \prime}$, and $\Theta^{\prime \prime}$, distinguishing them by two primes. The increments of these angles corresponding to a positive circuit of $\gamma$ are denoted by $\Delta \phi^{\prime \prime}, \ldots, \Delta \Theta^{\prime \prime}$.

The mapping $\Psi$ is conformal if $\left(x^{\prime}\right)^{2}+\left(y^{\prime}\right)^{2} \neq 0$ and reduces the polar angles to the vertex $x^{\prime}=y^{\prime}=0$ by the factor $|N|$. Thus,

$$
\begin{gathered}
\Delta \Theta=\Delta \Theta^{\prime}=\Delta \phi^{\prime}+\Delta \beta^{\prime}=|N| \Delta \beta^{\prime \prime}+\Delta \phi^{\prime \prime}, \\
\Delta \phi^{\prime \prime}<0, \quad \Delta \beta^{\prime \prime}=-\pi .
\end{gathered}
$$

Then , $-\pi>\Delta \Theta>\pi$. This is a contradiction. This completes the proof.

THEOREM 11. Suppose that $M$ is a complete, connected, orientable $C^{3}$ surface immersed in $R^{3}$ with a finite number of perfectly regular graph ends, and that $K<0$ in $R^{3}$. If the Gauss map converges to a point at infinity of each end, then $\chi(M) \leq 0$. 
Proof. By Lemma 10, there is no elliptic sector. Then, for each end $U_{i}$, along some Jordan curves looping around the end near infinity, we have

$$
L\left(U_{i}\right)=1+n_{e}^{i}-n_{h}^{i}=1-n_{h}^{i}
$$

where $e_{h}^{i} \geq 0$. Now, by Theorem 6 , we have

$$
2 \chi(M)=\sum\left(L\left(U_{i}\right)-1\right)=\sum-n_{h}^{i} \leq 0 .
$$

This completes the proof.

Therefore, we can have the following nonexistence corollary.

COROllary 12. There is no class $C^{3}$ graph $(x, y, f(x, y))$ over $R^{2}$ with $K<0$ whose Gauss map converges to a point at infinity and whose end is perfectly regular.

\section{§5. Application}

Theorem 1 may have applications to problems of visualization of twodimensional slices of solutions of the Einstein-Maxwell equation of gravitation and electromagnetism in source-free space during momentarily stationary symmetry on general relativity ([9], [1], [2]). Those two-dimensional slices can be considered as models of black holes. Any slice of the solutions of the Einstein-Maxwell equations of gravitation and electromagnetism in source-free space can be considered as a $C^{n}$-Riemannian surface $(n \geq 2)$ $M=\left(\Omega, d s^{2}\right)$, where $\Omega$ is a fundamental domain on the $u v$-plane $R^{2}$ and

$$
d s^{2}=E d u^{2}+2 F d u d v+G d v^{2}
$$

is the metric on $\Omega$. The fundamental domain indicates the region over $R^{2}$ and the topology. For example, the slice of the Schwarzschild solution is $M^{S c h}=\left(R^{2} \backslash\{(0,0)\}, d s^{2}\right)$, where

$$
d s^{2}=\left(1+\frac{m_{s}}{\sqrt{u^{2}+v^{2}}}\right)^{4}\left(d u^{2}+d v^{2}\right)
$$

and $m_{s}>0$. The Schwarzschild surface $M^{S c h}$ is a special case of the BrillLindguist (BL) surfaces such that $M^{S c h}=M_{l=1}^{B L}$. Let $M_{l}^{B L}=\left(R^{2} \backslash\left\{\left(u_{i}\right.\right.\right.$, $\left.\left.\left.v_{i}\right)\left.\right|_{i=1} ^{l}\right\}, d s_{l}^{2}\right)$, where

$$
d s_{l}^{2}=\phi_{l}^{4}\left\{d u^{2}+d v^{2}\right\}
$$


with

$$
\phi_{l}(u, v):=1+\sum_{i=1}^{i=l} \frac{m_{i}}{\sqrt{\left(u-u_{i}\right)^{2}+\left(v-v_{i}\right)^{2}}}
$$

where $l$ is a positive integer and $m_{i}>0$ is the mass. The topology is $\chi\left(M_{l}^{B L}\right)=1-l$.

Next, we define the Misner function as

$$
\varphi(u, v):=\sum_{n=-\infty}^{n=\infty} \frac{1}{\sqrt{\cosh (u+2 \mu n)-\cos v}}
$$

where $\mu$ is a nonzero constant. $\varphi(u, v)$ is a periodic function at $u$ and $v$.

Now, we define the Misner surface with multiply connected topology. Let $T_{1}=R^{2} / \Lambda$ be a torus with $\Lambda=2 \mu Z \oplus 2 \pi Z$ as the rectangular lattices. Let $\Omega_{1}=T_{1} \backslash\left\{p_{1}\right\}=\left\{(u, v) \in R^{2} \mid-\mu \leq u \leq \mu,-\pi \leq v \leq \pi\right\} \backslash\{(0,0)\}$ be the fundamental domain of $\varphi(u, v)$. Let $M_{1}^{M i s}=\left(\Omega_{1}, d s^{2}\right)$ be a Misner surface, where $d s^{2}=a^{2} \varphi^{4}\left\{d u^{2}+d v^{2}\right\}$ and $a$ is a nonzero constant. Then $M_{1}^{M i s}$ is homeomorphic to a one-point-punctured torus. Let $T_{2}=R^{2} / \Lambda$ and $\Lambda=4 \mu Z \oplus 2 \pi Z$ be another torus. Let $\Omega_{2}=T_{2} \backslash\left\{p_{1}, p_{2}\right\}=\left\{(u, v) \in R^{2} \mid\right.$ $-\mu \leq u \leq 3 \mu,-\pi \leq v \leq \pi\} \backslash\{(0,0),(2 \mu, 0)\}$ be another fundamental domain for $\varphi(x, y)$. Then $M_{2}^{M i s}=\left(\Omega_{2}, d s^{2}\right)$ is another abstract surface, and $M_{2}$ is homeomorphic to a two-points-punctured torus. In fact, $M_{2}^{M i s}$ is a doublecovering surface for $M_{1}^{M i s}$. We can extend the same idea to the surfaces $M_{l}^{\text {Mis }}, l>0$. We may call $M_{l}^{\text {Mis }}$ Misner surfaces, and the topology is $\chi\left(M_{l}^{M i s}\right)=-l$.

Misner surfaces $M_{l}^{\text {Mis }}(l>0)$ and BL surfaces $M_{l}^{B L}(l>0)$ have the following intrinsic properties [1].

(1) Their Gaussian curvatures are negative, and their total curvatures are finite, $\int|K| d A<\infty$.

(2) A closed geodesic curve loops around each singularity, and the end $U$ is defined as the region between the closed geodesic curve and the singularity. Thus, $\int_{U} K=-2 \pi$ for each end.

Also, for each end $K \rightarrow 0$ and the vanishing rate is the reciprocal of distance to the power 3 as points tend toward the singularity from all directions, so the intrinsic properties of the ends of Misner surfaces and BL surfaces are exactly the same.

A complete Schwarzschild surface $M^{S c h}$ can be $C^{2}$-isometrically embedded in $R^{3}$. Price and Romano [9] gave evidence by numerical computation 
that the Misner surface $M_{1}^{M i s}$ could not be isometrically embedded as a surface which, at large radius, had the paraboloidal shape of a standard embedding of Schwarzschild geometry. In [2], we discussed the explicit reasons, besides the Schwarzschild one, why other slices with a multiply connected topology cannot be visualized to any degree of completeness. The end of a Schwarzschild surface in $R^{3}$ is under the condition of $\int|B|^{2}<\infty$ extrinsically. Theorem 1 gives rigorous proof that rules out the possibility of $C^{2}$-isometrically embeddedness in $R^{3}$ of Misner surfaces and BL surfaces, except the embeddedness of the Schwarzschild surface because of the topological uniqueness.

\section{REFERENCES}

[1] H. Chan, Embedding Misner and Brill-Lindquist initial data for black-hole collisions, Math. Phys. Anal. Geom. 6 (2003), 9-27.

[2] H. Chan, Embedding negatively curved initial data of black-hole collisions in $R^{3}$, Classical Quantum Gravity 23 (2006), 225-234.

[3] H. Chan, Simply connected nonpositively curved surfaces in $R^{3}$, Pacific J. Math. 233 (2006), 1-4.

[4] H. Chan and A. Treibergs, Nonpositively curved surfaces, J. Differential Geom. 57 (2001), 389-407.

[5] C. Connell and M. Ghomi, Topology of negatively curved real affine algebraic surfaces, J. Reine Angew. Math. 624 (2008), 1-26.

[6] J. Hadamard, Les surfaces á courboures opposées et leurs lignes gé odésiques, J. Math. Pures Appl. 4 (1898), 27-73.

[7] P. Hartman, Ordinary Differential Equations, New York, Wiley, 1964, 27-73.

[8] F. Lopez and A. Ros, On embedded complete minimal surfaces of genus zero, J. Differential Geom. 33 (1991), 293-300.

[9] R. Price and J. Romano, Embedding initial data for black-hole collisions, Classical Quantum Gravity 12 (1995), 875-893.

[10] E. R. Rozendorn, Weakly irregular surfaces of negative curvature, Russian Math. Surveys 21 (1966), 57-112.

[11] R. Schoen, Uniqueness, symmetry, and embeddedness of minimal surfaces, J. Differential Geom. 18 (1983), 791-809.

[12] A. Verner, Topological structure of complete surfaces with nonpositive curvature which have one to one spherical mappings (in Russian), Vestnik Leningrad Univ. 20 (1965), $16-29$.

[13] A. Verner, Tapering saddle surfaces, Sib. Mat. Z. 11 (1968), 567-581.

[14] B. White, Complete surface of finite total curvature, J. Differential Geom. 26 (1987), $315-326$.

National Pingtung University of Education

Pingtung 900-03

Taiwan

hchan@mail.npue.edu.tw 